\title{
Criterios para la selección de alimentos de estudiantes universitarios de nutrición
}

\author{
María del Pilar Fernández-Carrasco *
}

\begin{tabular}{|c|c|}
\hline 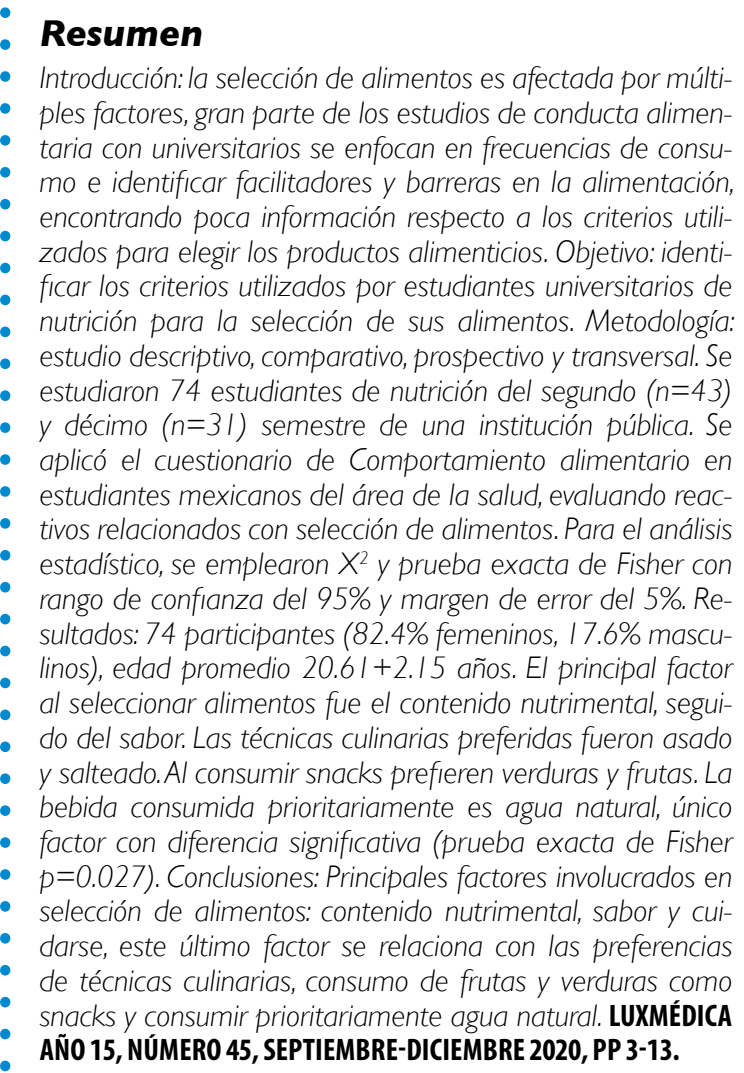 & 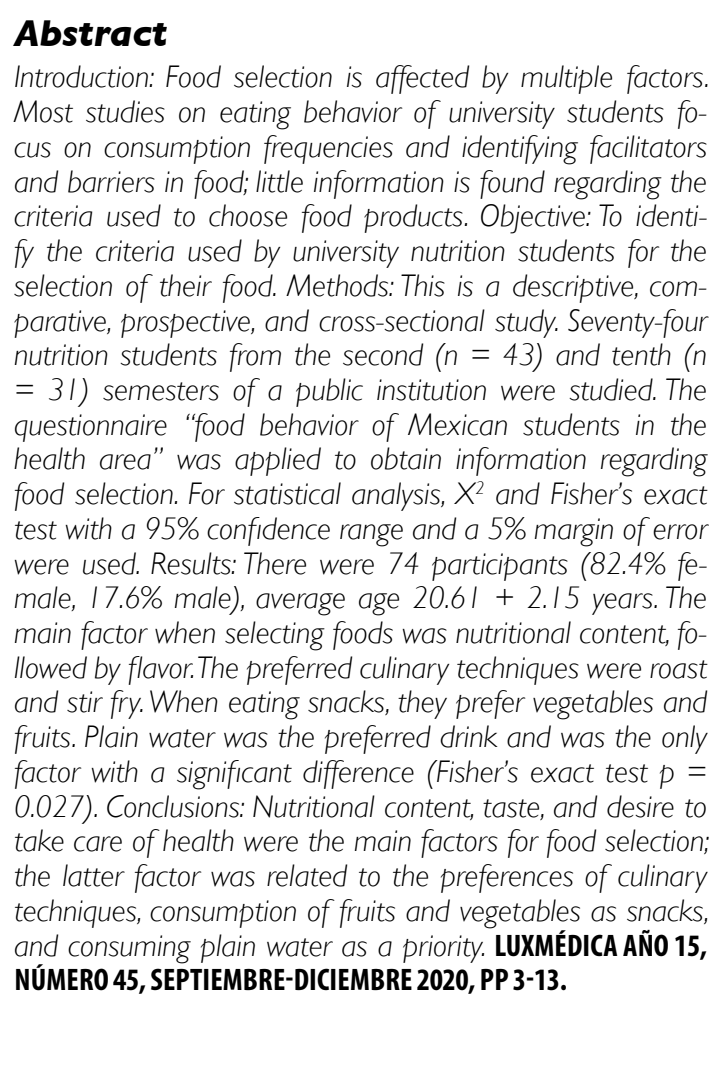 \\
\hline $\begin{array}{r}\text { Palabras clave: selección de alimentos, c } \\
\text { alimentario }\end{array}$ & $\begin{array}{l}\text { Keywords: food selection, food behavior, university } \\
\text { students }\end{array}$ \\
\hline
\end{tabular}

*Licenciada en Nutrición y Ciencias de los Alimentos. Maestra en Educación. Adscrita al Departamento de Medicina y Nutrición, División de Ciencias de la Salud, Universidad de Guanajuato Campus León.

Fecha de recibido: 11 de marzo 2020

Fecha de aceptación: 14 de julio 2020

Correspondencia. ME María del Pilar Fernández-Carrasco. Departamento de Medicina y Nutrición, División de Ciencias de la Salud, Universidad de Guanajuato, Campus León. Blvd. Puente Milenio \#1001. Fracción del Predio de San Carlos. Código postal 37670. León, Guanajuato, México. Teléfono 477-267-49-00 ext 3674 y 477-17-15-368. Orcid: 0000-0001-7480-0543 Correo electrónico mp.fernandez@ugto.mx 


\section{Introducción}

La Organización Mundial de la Salud (OMS) ha reportado que, en los países con bajos y medianos ingresos, las enfermedades no transmisibles (ENT) son responsables del $78 \%$ de las muertes, encontrándose entre las principales causas: la enfermedad isquémica del corazón, el infarto, la enfermedad obstructiva crónica, la diabetes mellitus y la cirrosis hepática, ${ }^{1}$ todas relacionadas con los malos hábitos del estilo de vida. Lo anterior se relaciona con los hallazgos epidemiológicos que vinculan a la alimentación y la nutrición con el desarrollo de patologías, ${ }^{2}$ entre ellas las ENT, por lo que en la medida en que se promueva la alimentación saludable se contribuirá a la disminución del desarrollo de éstas. ${ }^{3}$

En el caso de México, las modificaciones del estilo de vida de sus habitantes han contribuido a que en el país se establezca, desde hace varios años, una transición epidemiológica nutricional, responsable del incremento en el sobrepeso y obesidad, debida en cierta medida a la occidentalización de la dieta y el alto consumo de comida rápida, la cual incluye productos procesados con alto aporte de lípidos, hidratos de carbono y sal; la disminución del tiempo dedicado a la preparación de platillos y la mayor exposición a publicidad de alimentos, lo que, aunado a la disminución de actividad física, promueve el sedentarismo. ${ }^{4}$ Lo anterior permite comprender los resultados mostrados por la Encuesta Nacional de Salud y Nutrición en 2018 (ENSANUT 2018), la cual señala que la prevalencia combinada de sobrepeso y obesidad en adolescentes de entre 12 y 19 años incrementó 3.5\% respecto de la edición 2012 (38.4\% y $34.9 \%$, respectivamente) y que en adultos de 20 años y más, la prevalencia aumentó $3.7 \%$ ( $74.9 \%$ y $71.2 \%$, respectivamente), siendo más alta en el sexo femenino. ${ }^{5}$

La conducta alimentaria se refiere al comportamiento del individuo en relación con los alimentos, incluyendo la forma en que los selecciona, prepara y consume ${ }^{2}$ lo cual contribuye al estado de salud o enfermedad que manifiesta el sujeto. ${ }^{6}$ La alimentación es un acto voluntario y consciente que se ve influenciado por una serie de factores, ${ }^{2}$ como las características sociales, económicas y culturales de una población o región determinada. ${ }^{7}$

Como se señaló previamente, la selección de productos alimenticios se ve afectada por múltiples factores, los cuales pueden agruparse en: biológicos, los que afectan a los grupos sociales y los individuales. Los factores biológicos abarcan las características sensoriales de los alimentos (color, textura, sabor, olor, sonido que emiten al ser consumidos), los factores genéticos que condicionan déficits de enzimas asociados con intolerancias nutrimentales o que influyen en la sensibilidad gustativa para aceptar o rechazar ciertos alimentos y las necesidades nutricionales. Los factores que afectan a los grupos sociales abarcan las creencias, motivaciones, valores, costumbres, simbolismos, tradiciones, aspectos religiosos y factores económicos; en tanto que los factores individuales incluyen los as- 
pectos psicológico-afectivos y económicos, nivel educativo, conocimientos sobre alimentación y nutrición, socialización, actitudes, preferencias y rechazo de alimentos, entre otros. ${ }^{2}$

La transición de la adolescencia a la adultez conlleva transformaciones tanto biológicas como en el estilo de vida, coincidiendo esta etapa con el ingreso a la universidad, el vivir fuera del hogar familiar y buscar la independencia; convirtiéndola en un periodo crítico en lo referente a la salud ${ }^{8,9}$ por el riesgo de realizar prácticas nocivas recurrentes, como la alimentación inadecuada, el sedentarismo ${ }^{10}$ o el consumo de alcohol, así como el posible abandono de hábitos desarrollados durante la preparatoria al encontrarse ante una mayor libertad en la vida universitaria. ${ }^{11}$ La mayoría de los estudios con universitarios sobre comportamiento alimentario están enfocados hacia el conocimiento de sus hábitos alimentarios con énfasis en la frecuencia de consumo de alimentos, los facilitadores y las barreras en la alimentación, ${ }^{9-16}$ pero falta ampliar lo relacionado a los criterios de selección de alimentos. ${ }^{8}$ En la revisión de la literatura no se encontró una escala validada a nivel internacional para identificar los aspectos relacionados con la selección de alimentos, pero sí un instrumento validado en universitarios mexicanos que incluye preguntas relacionadas con la selección de alimentos. ${ }^{6}$ Con base en lo antes expuesto, el objetivo de esta investigación fue identificar los criterios utilizados por estudiantes universitarios de nutrición para la selección de sus alimentos.

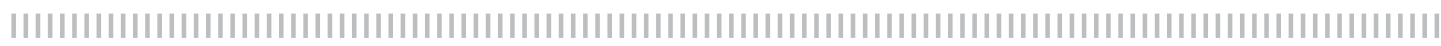

\section{Material y métodos}

Se realizó un estudio descriptivo, comparativo, prospectivo y de corte transversal, durante el semestre enero-junio 2018, con estudiantes universitarios de nutrición de una institución pública, con la finalidad de identificar los criterios que emplean para seleccionar sus alimentos. Debido al momento en que se realizó el estudio y la admisión anual al programa educativo, la población de estudio fue de 74 sujetos, considerando a los estudiantes que habían ingresado más recientemente al programa educativo (segundo semestre, $n=43$ ) y aquellos próximos a concluir sus estudios (décimo semestre, $\mathrm{n}=31$ ). Los criterios de inclusión consideraron a los alumnos de los semestres y programa antes mencionados en el periodo señalado y que aceptaran la participación voluntaria. Se consideraron como criterios de exclusión estudiantes de otros programas educativos y semestres, así como aquellos que no completaron el cuestionario o presentaron embarazo. La muestra final fue de 74 estudiantes.

Se utilizó un formato autoadministrado de elaboración propia para obtener información general (sexo, edad, licenciatura, semestre y presencia o no de embarazo) junto con el cuestionario validado, Comportamiento alimentario en estudiantes mexicanos del área de la salud de Márquez-Sandoval. ${ }^{6} \mathrm{El}$ instrumento tiene alfa de Cronbach 0.98 y consta de 31 preguntas con opción de respuesta múltiple con escala tipo Likert para evaluar 4 factores: selección de alimentos (ítems: 1, 3, 4, 23 y 24), prácticas y habilidades personales (ítems: $2,5,7,8,9,10,25,26,28$ y 31), autopercepción de la alimentación $(6,27,29$ y 30) y agrado hacia alimentos $(11,12,13,14$, $15,16,17,18,19,20,21$ y 22). Con base 
en la literatura, los autores calificaron cada opción de respuesta como "saludable" (1 punto) o "no saludable" (0 puntos), en algunos ítems consideraron más de una respuesta como saludable. Para la presente investigación, se evaluó el factor de selección de alimentos con la finalidad de identificar si los criterios que emplean los estudiantes para tal fin se modifican de acuerdo con el tiempo que tienen de formación académica en el área de nutrición, es decir, los semestres de estudio cursados.

Para responder el cuestionario se proporcionó un tanto impreso a cada participante y la información recabada fue capturada en
Excel. Se llevó a cabo un análisis estadístico descriptivo (media y desviación estándar) para los datos generales e inferencial $\left(X^{2} y\right.$ prueba exacta de Fisher) para los criterios de selección de alimentos. Se consideró una $p<0.05$ estadísticamente significativa. Los datos fueron procesados estadísticamente con el programa SPSS versión 23.0. El protocolo de este estudio consideró los criterios de la Declaración de Helsinki ${ }^{17}$ y antes de comenzar el estudio se explicó a los participantes la naturaleza del proyecto, sus objetivos y beneficios; a quien voluntariamente aceptó participar, se le solicitó el llenado del consentimiento informado.

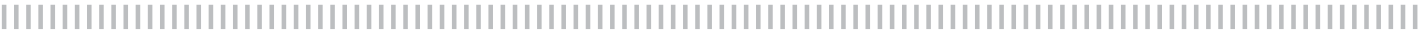

\section{Resultados}

No se eliminaron cuestionarios por llenado incompleto ni por embarazo. Participaron 74 estudiantes de los cuales 43 cursaban el segundo semestre y 31 , el décimo $(58.1 \%$ y $41.9 \%$, respectivamente). La edad promedio fue $20.61 \pm 2.15$ años (rango: 18-27), siendo 61 del sexo femenino y 13 del mas- culino ( $82.4 \%$ y $17.6 \%$, respectivamente).

Para el factor de selección de alimentos se obtuvo el promedio de cada ítem por semestre (tabla 1) y se realizó la comparación entre los mismos mediante $x^{2}$ y prueba exacta de Fisher, encontrando con esta última un valor significativo $(p=0.027)$ para la elección de bebidas (tabla 2).

\section{Tabla}

Puntuaciones de los ítems del factor "selección de alimentos por semestre"

\begin{tabular}{|lcc|}
\hline Ítem & Segundo semestre & Décimo semestre \\
\hline & $(\mathrm{n}=43)$ & $(\mathrm{n}=31)$ \\
\hline Factores más importantes al seleccionar alimento & $3.37+1.86$ & $3.58+1.94$ \\
\hline Motivos para evitar alimentos & $1.76+0.84$ & $1.48+0.72$ \\
\hline Técnica de preparación de alimentos & $3.34+1.19$ & $3.54+1.17$ \\
\hline Bebidas seleccionadas & $2.07+0.33$ & $1.90+0.39$ \\
\hline Preferencias de consumo de snacks & $2.53+1.46$ & $2.93+1.80$ \\
\hline
\end{tabular}

Datos mostrados en media y desviación estándar $(n=74)$. 


\section{Tabla 2}

Pruebas estadísticas empleadas para la comparación de ítems del factor "selección de alimentos por semestre"

\begin{tabular}{|c|c|c|}
\hline Ítem & Chi cuadrada & Prueba exacta de Fisher \\
\hline Factores más importantes al seleccionar alimento & $\begin{array}{l}X^{2}=4.893 \\
p=0.298\end{array}$ & $\begin{array}{c}\text { P. Fisher }=4.062 \\
p=0.372\end{array}$ \\
\hline Motivos para evitar alimentos & $\begin{array}{l}x^{2}=2.822 \\
p=0.420\end{array}$ & $\begin{array}{c}\text { P. Fisher }=2.831 \\
p=0.480\end{array}$ \\
\hline Técnica de preparación de alimentos & $\begin{aligned} X^{2} & =1.710 \\
p & =0.635\end{aligned}$ & $\begin{array}{c}\text { P. Fisher }=1.926 \\
p=0.626\end{array}$ \\
\hline Bebidas seleccionadas & $\begin{array}{l}X^{2}=6.585 \\
p=0.086\end{array}$ & $\begin{array}{l}\text { P. Fisher }=6.372 \\
\mathbf{p}=\mathbf{0 . 0 2 7}\end{array}$ \\
\hline Preferencias de consumo de snacks & $\begin{array}{l}X^{2}=7.489 \\
p=0.187\end{array}$ & $\begin{array}{c}\text { P. Fisher }=7.111 \\
p=0.142\end{array}$ \\
\hline
\end{tabular}

$p=$ valor de $p ;{ }^{*} p<0.05$ prueba exacta de Fisher para bebidas seleccionadas $(n=74)$.

Respecto a los ítems analizados, el principal factor para la selección de alimentos en ambos semestres fue el contenido nutrimental con $51.2 \%$ para segundo y $64.5 \%$ para décimo. Este aspecto es la respuesta saludable para los autores del instrumen- to. ${ }^{6}$ Llama la atención que los estudiantes de segundo semestre presentaron mayor variedad en las opciones de respuesta, ya que incluyeron la caducidad $(7 \%)$, que el alimento fuera agradable a la vista $(4.7 \%)$ y el precio (2.3\%) (figura 1 ).

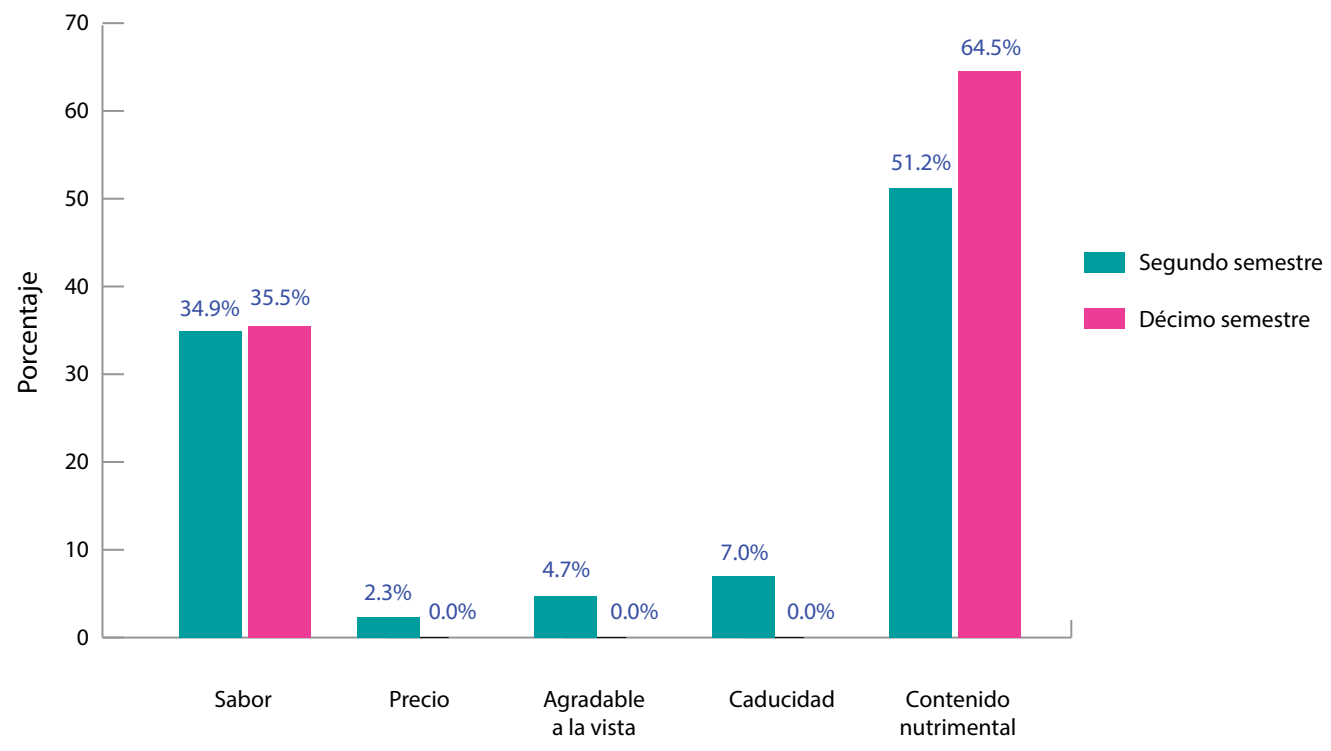

Figura 1. Se muestran los factores más importantes al seleccionar alimentos entre estudiantes de nutrición $(n=74)$. No se encontraron diferencias significativas entre las respuestas por semestre al realizar la prueba de X2 ( $p=0.298)$ ni la prueba exacta de Fisher ( $p=0.372)$. 
Respecto a los motivos para evitar el consumo de algún alimento, la respuesta con mayor porcentaje en ambos semestres fue el no agrado por el producto (41.9\% y $63.3 \%$, respectivamente), siendo mayor en los alumnos de último semestre. El siguiente factor fue el autocuidado, con un mayor porcentaje para segundo que para décimo semestre $(46.5 \%$ y $32.3 \%$, respectivamente), siendo ésta la respuesta que, a juicio de los autores del instrumento, es la respuesta saludable. ${ }^{6}$ En contraparte, el $7 \%$ de los alumnos del segundo semestre y el $3.2 \%$ de los de décimo refirieron que no evitan alimentos (figura 2 ).

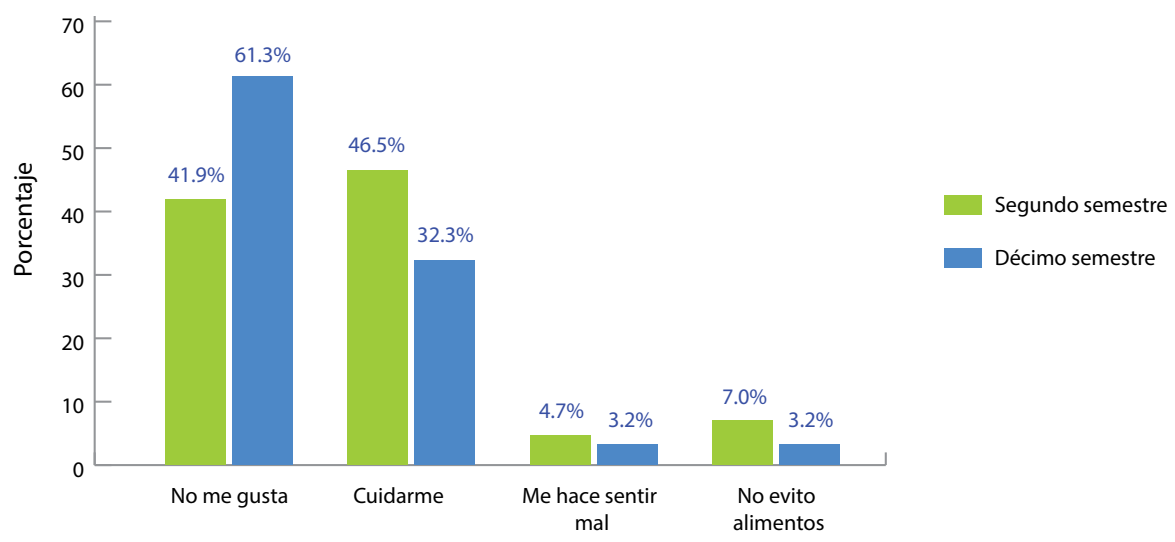

Figura 2. Se indican los motivos para evitar alimentos entre estudiantes nutrición $(n=74)$. No se encontraron diferencias significativas entre las respuestas por semestre al realizar la prueba de $X^{2}(p=0.420)$ ni la prueba exacta de Fisher $(p=0.480)$.

Otro criterio para la elección de los alimentos es la técnica culinaria empleada en su preparación. En este sentido, el mayor porcentaje para ambos semestres es el asado ( $46.5 \%$ y $51.6 \%$, respectivamente), considerado como una de las dos posibilidades de respuesta saludable para los autores; la otra opción fue la técnica al vapor. ${ }^{6}$ Se destaca que la fritura o freído fue la técnica menos preferida en ambos grupos $(2.3 \%$ y $3.2 \%$, respectivamente) (figura 3).

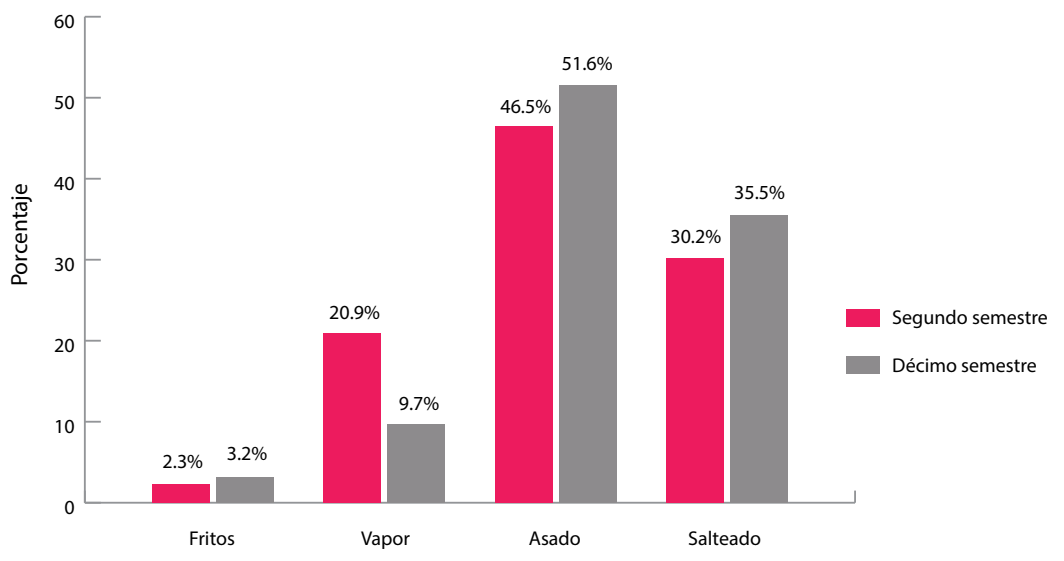

Figura 3. Técnicas de preparación de alimentos seleccionadas entre estudiantes de nutrición (n=74). No se encontraron diferencias significativas entre las respuestas por semestre al realizar la prueba de $X 2$ ( $p=0.635)$ ni la prueba exacta de Fisher ( $p=0.626$ ). 
La alimentación incluye las bebidas que se ingieren, en este aspecto los alumnos de ambos semestres prefieren prioritariamente, a lo largo del día, el consumo de agua natural, siendo mayor el porcentaje en el caso de los estudiantes de segundo (95.3\%) que para los de décimo (83.9\%); ésta es la respuesta considerada saludable en el instrumento. ${ }^{6}$ El menor porcentaje de participantes bebe leche durante el día (2.3\% alumnos de segundo semestre y ninguno del décimo) (figura 4).
Es frecuente que, a lo largo del día, se realicen colaciones y se consuman snacks entre comidas. En este sentido, gran parte de los participantes seleccionan frutas o verduras, siendo mayor el porcentaje para los estudiantes de segundo semestre que para los de décimo (83.7\% y $61.3 \%)$, el instrumento indica que ésta es la respuesta saludable. ${ }^{6}$ Solamente el $4.7 \%$ de los alumnos de segundo y el $3.2 \%$ de los de décimo refirieron no ingerir alimentos entre comidas (figura 5).

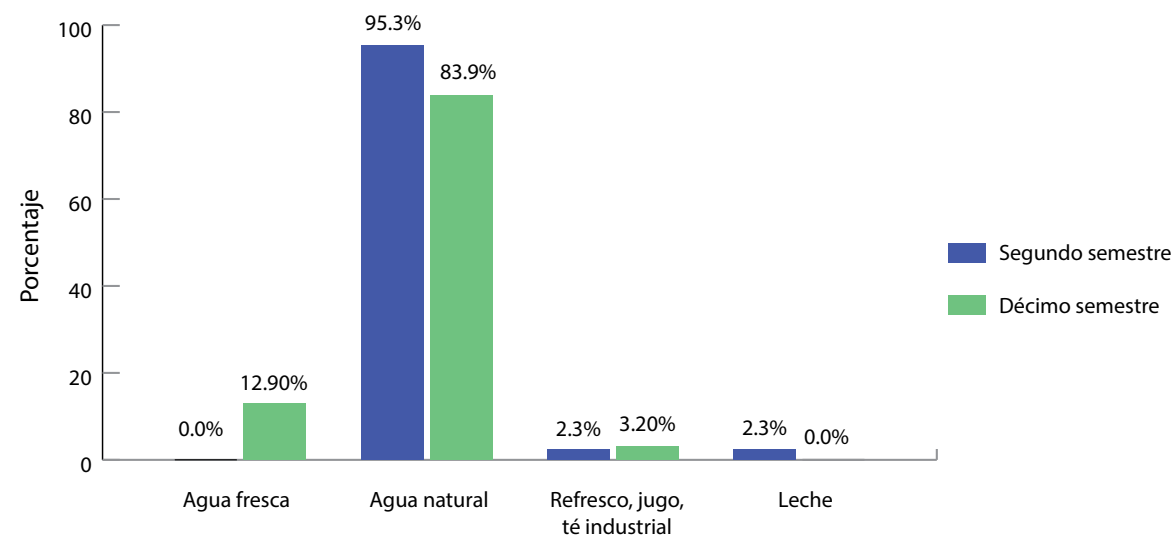

Figura 4. Se muestran las bebidas seleccionadas entre estudiantes de nutrición (n=74). Se encontró diferencia significativa entre las respuestas por semestre al realizar la prueba exacta de Fisher ( $p=0.027)$.

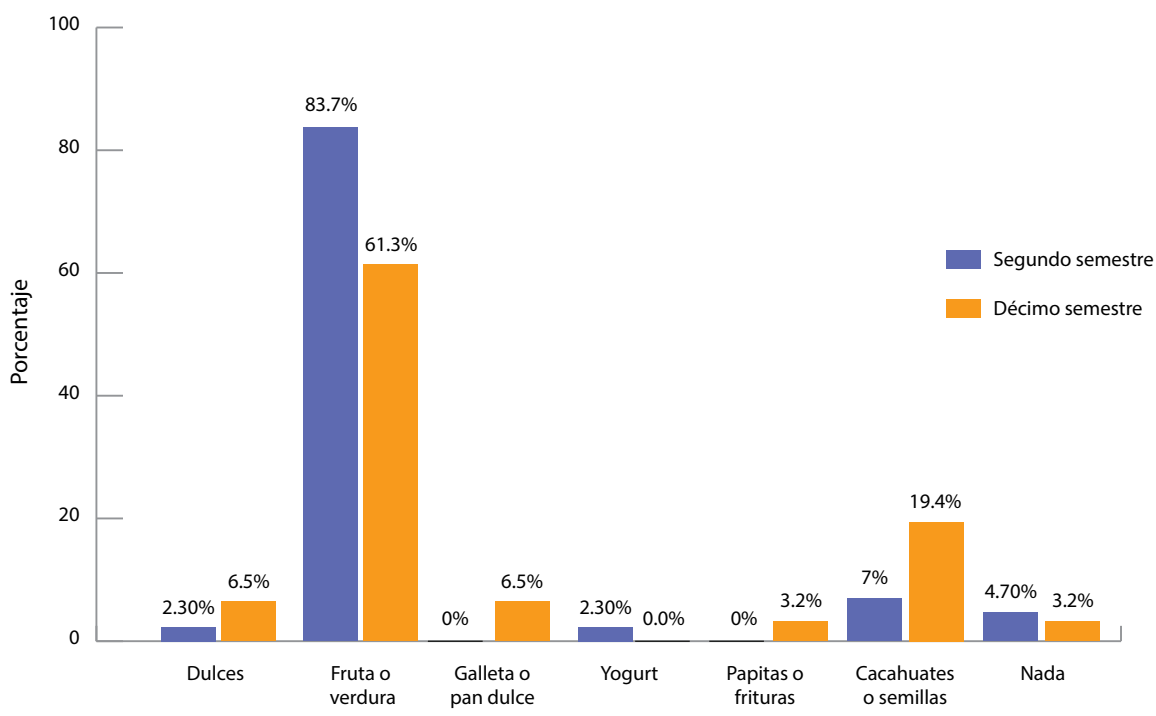

Figura 5. Se indica la selección para el consumo de snacks entre estudiantes de nutrición ( $n=74)$. No se encontraron diferencias significativas entre las respuestas por semestre al realizar la prueba de $X^{2}(p=0.187)$ ni la prueba exacta de Fisher ( $p=0.142$ ). 


\section{Discusión}

En esta investigación predominó la participación del sexo femenino debido a que el $82.4 \%$ de la matrícula del programa educativo es femenina, esta situación es similar a lo reportado por otros estudios realizados con alumnos de esta disciplina. ${ }^{18,19}$

Es importante señalar que no se encontró una escala internacional o nacional exclusiva para identificar los criterios para la selección de alimentos; sin embargo, existe una gran variedad de instrumentos para evaluar el comportamiento alimentario que han sido elaborados para atender intereses particulares, por lo que sus hallazgos suelen ser específicos para sus poblaciones de estudio, como es el caso de la practicidad para preparar y/o consumir los alimentos. $8,13,14,20$

El objetivo del presente estudio fue identificar los criterios involucrados en la selección de alimentos sin priorizar que las respuestas obtenidas fueran las que el instrumento empleado consideraba saludables; sin embargo, al cotejar la información, se encontró que el mayor porcentaje de respuestas coincidía con lo esperado, exceptuando el ítem sobre los motivos para evitar alimentos, en el que el $40 \%$ de los sujetos $(46.5 \%$ del segundo semestre y $32.3 \%$ del décimo) señaló el cuidarse (respuesta saludable) y el 50\% (41.9\% y $61.3 \%$ respectivamente) indicó como principal razón para no consumir un alimento que no les gustaba (respuesta no saludable). Si bien los porcentajes son similares, se refuerza que el sabor es un aspecto importante al consumir productos alimenticios. Lo anterior puede deberse a que su formación académica les permite identificar sustitutos para los alimentos que no les agradan, sin descuidar el contenido nutrimental, ya que el $59.5 \%(51.2 \%$ y $64 \%$ respectivamente) señaló este último criterio como el más importante. La mayoría de las investigaciones se centran en los alimentos consumidos ${ }^{9-11,15,16}$ sin reportar las razones para evitarlos, por lo que se detec- ta la necesidad de ahondar en las mismas.

En la literatura es ampliamente señalado que el sabor es el indicador más relevante para consumir un alimento ${ }^{8,14,20-23}$ y el precio 9,12,13,21,24 el segundo; a pesar de ello, en esta investigación solamente un participante señaló el costo. Lo anterior puede deberse a que en México convergen dos aspectos relevantes para la producción de alimentos: su clima y la biodiversidad de especies, lo que favorece la producción de una gran variedad a bajo costo, como es el caso de frutas y verduras, las cuales suelen tener un precio elevado en otros países. ${ }^{25,26}$

En lo referente a las técnicas culinarias para la preparación de alimentos, se encontró que se seleccionan prioritariamente platillos cocinados al vapor o salteado y que solamente $2.8 \%$ de los participantes prefiere el freído. Varios estudios reportan frecuencias de consumo con un apartado para comida chatarra, ${ }^{16}$ comida rápida ${ }^{9} 0$ alimentos fritos, ${ }^{15,16}$ indicando que hasta el $35 \%$ de los sujetos consume estos últimos de 1 a 3 veces por semana, sin identificar la forma de preparación del resto de los alimentos. Una investigación con universitarios onubenses encontró que pocos estudiantes saben cocinar saludablemente o prefieren no hacerlo, ${ }^{10}$ situación que es diferente a la de la población del presente estudio, porque desde los primeros semestres de formación cursan asignaturas relacionadas con la selección y preparación de alimentos.

La dieta abarca los platillos y bebidas ingeridos. En la selección de éstas se encontró valor estadísticamente significativo $(p<0.027)$ para el agua natural, lo que coincide con otros estudios en los que se refiere que es la preferida por los universitarios para hidratarse, con una frecuencia que va del $65 \%{ }^{15}$ al $93.8 \% .{ }^{10}$ En contraparte, del $10.3 \% 10$ al $30 \%{ }^{15}$ beben refres$\mathrm{CO}$, aunque en el presente trabajo fue aún más bajo $(2.7 \%)$. Si bien la literatura refiere que el consumo de alcohol varias veces a la semana es frecuente entre estudiantes 
de pregrado, 11,15,16 éste no fue referido por los participantes, lo que se explica dado que el ítem del cuestionario enfatizaba la bebida seleccionada prioritariamente durante el día.

Se identificó que el $74.3 \%$ de sujetos prefirió las frutas o verduras como snack, seguido de cacahuates $u$ otras semillas $(12.2 \%)$ y dulces $(4.1 \%)$, entre las diferencias por semestre se encontró que los alumnos del segundo semestre prefieren el yogurt y los del décimo las galletas o pan dulce y las "papitas" o frituras, lo que, si bien se presenta en bajo porcentaje, llama la atención y puede deberse a que se ha observado que los estudiantes de los primeros semestres suelen tratar de cumplir la expectativa social de que los nutriólogos solamente consumen productos saludables $y$ al ir avanzando en su formación académica se dan cuenta que en la dieta pueden incluirse diferentes alimentos, cobrando más relevancia el tamaño de las porciones. La respuesta de frutas y verduras puede explicarse porque en México existe una gran variedad de éstas a bajo costo y el tiempo que requieren para su preparación (lavar, desinfectar, pelar) es relativamente pequeño, ya que pueden disfrutarse crudas.
Lo anterior se relaciona con estudios que señalan el costo de los alimentos, 9,12,13,21,24 el tiempo para prepararlos $\mathrm{y} / \mathrm{o}$ consumir$\operatorname{los}^{13,22,25}$ y la conveniencia ${ }^{14,20}$ como aspectos importantes para su selección. Adicionalmente, tienen bajo aporte energético y su elección puede contribuir a reforzar el autocuidado, ${ }^{12,24}$ aspecto que, en este estudio, el $40 \%$ de los participantes señaló que lo hacía para evitar consumir alimentos. En un estudio con universitarios alemanes se encontró que las opciones de snacks consumidos estaban conformadas por chocolate, pasteles y galletas, helado, gomitas, comida rápida y papas fritas, ${ }^{9}$ lo que puede indicar que la disponibilidad de alimentos en los países al ser diferente puede influir, entre otros aspectos, en las preferencias para seleccionarlos. ${ }^{23}$

En general, los hallazgos de este estudio coinciden con la evidencia de que la selección de alimentos es el resultado de decisiones complejas, individuales e influenciadas por muchos factores. ${ }^{8,23} \mathrm{Si}$ bien el presente trabajo no es exhaustivo, permite vislumbrar los aspectos considerados por los jóvenes al momento de elegir su comida, ya que existen pocos estudios en este sentido.

\section{Conclusiones}

Los criterios para la selección de alimentos son individuales e influenciados por varios aspectos. En la población universitaria estudiada, los principales factores involucrados fueron el contenido nutrimental, el sabor y cuidarse, este último factor se corrobora con las preferencias de técnicas culinarias de asado y salteado, ingesta de frutas y verduras como snacks y consumo de agua natural. A diferencia de otros estudios, el precio no fue un criterio relevante para la elección de alimentos. Se destaca que un gran porcentaje de las respuestas brindadas por los participantes son consideradas saludables, lo cual puede deberse a la formación académica que reciben. 


\section{Sugerencias}

Se recomienda realizar estudios complementarios enfatizando las razones por las que los universitarios evitan consumir ciertos alimentos, así como incluir las técnicas culinarias empleadas, lo anterior podría derivar en la generación de un instrumento homologado por región o país que facilite la comparación de los hallazgos y el establecimiento de estrategias educativas como una medida para contrarrestar la prevalencia de sobrepeso y obesidad en los adultos mexicanos.

\section{Conflicto de interés}

Los autores declaran no tener conflicto de interés.

\section{Bibliografía}

\section{| | | | | | | | | | | | | | | | | | | | | | | | | | | | | | | | | | | | | | | | | | | | | | | | | | | | | | | | | | | | | | | | | | | | | | | | | | | | | | | | | | | | | | | | | | | | | | | | | | | | ||}

1. Organización Mundial de la Salud. Las 10 principales causas de defunción. Notas descriptivas. [Internet]. Organización Mundial de la Salud; 2018 [citado 20 octubre 2019]. Disponible en: https://www. who.int/es/news-room/fact-sheets/detail/the-top10-causes-of-death

2. Aranceta Bartrina J. Preferencias y hábitos alimentarios. En: Nutrición comunitaria. 3a ed. Barcelona, España: Elsevier Masson; 2013. p. 215-29.

3. World Health Organization. Healthy Diet. [Internet] Healthy diet. Fact sheets. 2018 [consultado 25 de diciembre de 2019]. Fact Sheet: Healthy Diet. Disponible en: https://www.who.int/news-room/factsheets/detail/healthy-diet

4. Secretaría de Salud. Estrategia Nacional para la prevención y el control del Sobrepeso, la Obesidad y la Diabetes. [Internet]. Ciudad de México: Instituto NAcional de Salud Pública; 2016 [citado 12 octubre 2019]. Disponible en: https://www.gob.mx/cms/ uploads/attachment/file/276108/estrategia_sobrepeso_diabetes_obesidad.pdf

5. Instituto Nacional de Salud Pública. Encuesta Nacional de Salud y Nutrición de 2018 (ENSANUT 2018) Presentación de resultados. [Internet]. México; 2018. Disponible en: https://ensanut.insp.mx/encuestas/ ensanut2018/doctos/informes/ensanut_2018_presentacion_resultados.pdf

6. Márquez-Sandoval YF, Salazar-Ruiz EN, MacedoOjeda G, Altamirano-Martínez MB, Bernal-Orozco MF, Salas-Salvadó J, et al. Diseño y validación de un cuestionario para evaluar el comportamiento alimentario en estudiantes mexicanos del área de la salud. Nutr Hosp. [Internet] 2014 [citado 1 octubre 2019] 30(1): p.153-164 Disponible en: http://scielo.isciii. es/pdf/nh/v30n1/20originalvaloracionnutricion al01.pdf

7. Secretaría de Gobernación. Norma Oficial Mexicana NOM-043-SSA2-2012. Servicios básicos de salud. Promoción y educación para la salud en materia alimentaria. Criterios para brindar orientación. Diario Oficial de la Federación (22-02-2013).
8. Stok FM, Renner $B$, Clarys $P$, Lien $N$, Lakerveld $\mathrm{J}$, Deliens T. Understanding Eating Behavior during the Transition from Adolescence to Young Adulthood?: A Literature. Nutrients [Internet]. 2018 [citado 1 noviembre 2019];10(667):1-16. Disponible en: https://www.ncbi.nlm.nih.gov/pmc/articles/ PMC6024552/pdf/nutrients-10-00667.pdf

9. Hilger-Kolb J, Loerbroks A, Diehl K. Eating behaviour of university students in Germany: Dietary intake, barriers to healthy eating and changes in eating behaviour since the time of matriculation. Appetite [Internet]. 2017[citado 3 noviembre 2019]; 109:100-7. Disponible en: https://www.researchgate.net/ publication/310437216_Eating_behaviour_of_university_students_in_Germany_Dietary_intake_barriers_to_healthy_eating_and_changes_in_eating behaviour_since_the_time_of_matriculation

10. López G, Sosa $\bar{E}$, Garrido $A$, Travé G GF. Hábitos , preferencias y habilidades culinarias de primer curso de la universidad de Huelva. Enfermería Global [Internet]. 2019 [citado 1 noviembre 2019];18(3):12741. Disponible en: https://dialnet.unirioja.es/servlet/articulo?codigo $=6992888$

11. Deforche B, Van Dyck D, Deliens T, De, Bourdeaudhuij I. Changes in weight, physical activity, sedentary behaviour and dietary intake during the transition to higher education?: a prospective study. Int J Behav Nutr Phys Act [Internet]. 2015 [citado 19 noviembre 2019];12(16):1-10. Disponible en: https:// ijbnpa.biomedcentral.com/track/pdf/10.1186/ s12966-015-0173-9

12. Munt $A$, Partridge $S$, Allman-Farinelli $M$. The barriers and enablers of healthy eating among young adults?: a missing piece of the obesity puzzle?: A scoping review . Obes Rev. 2017;18(1):1-17.

13. Duarte-Cuervo CY, Ramos-Caballero DM, LatorreGuapo ÁC, González-Robayo PN. Factores relacionados con las prácticas alimentarias de estudiantes de tres universidades de Bogotá Factors. Rev salud pública [Internet]. 2015 [citado 1 diciembre 2019];17(176):925-37. Disponible en: http:// dx.doi.org/10.15446/rsap.v17n6.38368 
14. Tam R, Yassa $B$, Parker $\mathrm{H}$, Connor $\mathrm{OH}$. University students' on-campus food purchasing behaviors, preferences, and opinions on food availability. Nutrition [Internet]. 2017[citado 1 noviembre 2019]; May;37:7-13. Disponible en: https://www. sciencedirect.com/science/article/abs/pii/ S0899900716301277

15. Pereira A, Doumid A, Bezerra K, Bandeira G, Conter L. Perfil e hábitos alimentares de acadêmicos ingressantes do curso de nutrição de uma universidade do sul do brasil. Rev Bras Obesidade, Nutr e Emagrecimento [Internet]. 2018 [citado 1 noviembre 2019];12(75):859-66. Disponible en: http://web.b.ebscohost.com/ehost/pdfviewer/ pdfviewer?vid=17\&sid=77367ad6-46f6-41458cf6-30d897ef3786\% 40pdc-v-sessmgr03

16. Durán $S$, Crovetto $M$, Espinoza $V$, Mena $F$, Oñate G, Fernández M. Caracterización del estado nutricional, hábitos alimentarios y estilos de vida de estudiantes universitarios chilenos: estudio multicéntrico. Rev Med Chile [Internet]. 2017 [citado 7 noviembre 2019];145(11):1403-11. Disponible en: https://scielo.conicyt.cl/scielo.php?script $=$ sci arttext\&pid =S0034-98872017001101403\&lng =es

17. Asociación Médica Mundial. Declaración de Helsinki de la AMM. Principios éticos para las investigaciones médicas en seres humanos [Internet]. 2017 [citado 1 octubre 2019]; p. 1-5. Disponible en:: https://www. wma.net/es/policies-post/declaracion-de-helsinkide-la-amm-principios-eticos-para-las-investigaciones-medicas-en-seres-humanos/

18. Rocks T, Pelly F, Slater G, Martin LA. Eating attitudes and behaviours of students enrolled in undergraduate nutrition and dietetics degrees. Nutr Diet [Internet]. 2017 [citado 5 noviembre 2019];74:381-7. Disponible en: http://web.b.ebscohost.com/ehost/ pdfviewer/pdfviewer?vid $=27 \&$ sid $=77367$ ad6 46f6-4145-8cf6-30d897ef3786\% 40pdc-v-sessmgr03

19. Herrada-Lemus J, Casillas-Bonilla A, García-Magdaleno D, Ramírez-Martínez $M$, Reyes-Ruiz Esparza I, Campo-Cervantes $M$ del, et al. Hábitos alimenticios en estudiantes de nutrición de la Universidad Autónoma de Aguascalientes en el periodo enero-junio 2015. Lux Médica [Internet]. 2016; [citado 6 noviembre 2019]; (33):3-8. Disponible en: https:// revistas.uaa. $\mathrm{mx} /$ index.php/luxmedica/article/viewFile/720/697
20. De Oliveira Lima-Filho D, Quevedo-Silva F, Gomes Coehl, Ewerling S, Rodrigues da Silva W. Criterios de selección y de consumo alimentarios en pequeñas ciudades de Brasil. Invenio [Internet]. 2016 [citado 8 noviembre 2019];19(37):123-35. Disponible en: https://www.redalyc.org/pdf/877/87747436008. pdf

21. Farfán M, Olarte G. Prevalencia de sobrepeso , obesidad, comportamientos alimentarios y de actividad fisica en estudiantes Universitarios - Boyacá 2013 Prevalence of overweight, obesity, behaviour food and physical activity. Rev Investig Salud Univ Boyacá [Internet]. 2014 [citado 9 noviembre 2019];1(2):204-16. Disponible en: http://revistasdigitales.uniboyaca.edu.co/index.php/rs/article/ view/123

22. Deliens T, Clarys P, De Bourdeaudhuij I, Deforche B. Determinants of eating behaviour in university students: a qualitative study using focus group discussions. Discovery Service for Endeavour College of Natural Health Library. BMC Public Health [Internet]. 2014 [citado 1 noviembre 2019];14(53):1-22. Disponible en: http://www.biomedcentral.com/14712458/14/53\% OARESEARCH

23. Sogari G, Velez-Argumedo C, Miguel IG, Mora C. College Students and Eating Habits?: A Study Using An Ecological Model for Healthy Behavior. Nutrients [Internet]. 2018[citado 3 noviembre 2019];10(12):1-16. Disponible en: https://www. ncbi.nlm.nih.gov/pmc/articles/PMC6315356/

24. Howse E, HAnkey C, Allman-Farinelli $M$, Bauman A, Freeman B. 'Buying Salad Is a Lot More Expensive than Going to McDonalds': Young Adults' Views about What Influences Their Food Choices. Nutrients [Internet]. 2018 [citado 11 noviembre 2019];10(996):6. Disponible en: https://www.ncbi. nlm.nih.gov/pmc/articles/PMC6116192/pdf/nutrients-10-00996.pdf

25. Venn D, Strazdins L. Your money or your time?? How both types of scarcity matter to physical activity and healthy. Soc Sci Med 2017;172:98-106.

26. ?akar U, ?obaji? S, Vidovi? B, Djordjevi? B. Nutritional and lifestyle habits of European pharmacy undergraduate students. Progr Nutr. 2018;20(1):38-45. Disponible en: https://mattioli1885journals.com/ index.php/progressinnutrition/article/view/5435 